\title{
Chemometric evaluation of the column classification system during the pharmaceutical analysis of lamotrigine and its related substances
}

\author{
Jarosław Szulfer • Alina Plenis • Tomasz Bączek
}

Received: 15 April 2013 /Revised: 23 May 2013 / Accepted: 27 May 2013 / Published online: 28 June 2013

(C) The Author(s) 2013. This article is published with open access at Springerlink.com

\begin{abstract}
This paper investigates the performance of a column classification system developed at the Katholieke Universiteit Leuven applied to pharmaceutical chromatographic analyses. The liquid chromatography assay of lamotrigine and related compounds was carried out according to the method prescribed in the European Pharmacopoeia monograph, using 28 brands of stationary phases. A ranking was built based on the $F_{\text {KUL }}$ value calculated against the selected reference column, then compared with the column test performance established for the stationary phases studied. Therefore, the system suitability test prescribed by the European Pharmacopoeia in order to distinguish between suitable or unsuitable columns for this analysis was evaluated. Moreover, it was examined whether the classes of the stationary phases, determined using test parameter results, contain either suitable or unsuitable supports for the lamotrigine separation. This assay was performed using chemometric a technique, namely factor analysis.
\end{abstract}

Keywords Column classification systems · The Katholieke Universiteit Leuven method · High-performance liquid chromatography $\cdot$ Factor analysis $\cdot$ Lamotrigine and its related substances

\section{Introduction}

Nowadays, a very large number of different brands of reversedphase liquid chromatography (RP-LC) stationary phases are

\footnotetext{
J. Szulfer

Polpharma SA Pharmaceutical Works, Pelplińska 19,

83-200 Starogard Gdański, Poland
}

\author{
A. Plenis · T. Bączek $(\triangle)$ \\ Department of Pharmaceutical Chemistry, Medical University of \\ Gdańsk, Hallera 107, \\ 80-416 Gdańsk, Poland \\ e-mail: tbaczek@amg.gda.pl
}

commercially available on the market and new ones are being introduced regularly [1]. It means that the selection of a RP-LC column with suitable selectivity for a particular separation is difficult [2]. Many papers describing methods of characterizing columns were published to solve this issue [3-14], while others are under development $[15,16]$. An interesting procedure of column selectivity of RP-LC $\mathrm{C}_{18}$ stationary phases, namely the Katholieke Universiteit Leuven method (KUL method), has been provided by the group of Hoogmartens and co-workers $[17,18]$. In this approach, each $\mathrm{C}_{18}$ column is characterized by four chromatographic parameters: the retention factor of amylbenzene $\left(k_{\mathrm{amb}}^{\prime}\right)$ reflecting hydrophobicity, the relative retention factor of benzylamine/phenol at $\mathrm{pH} 2.7$

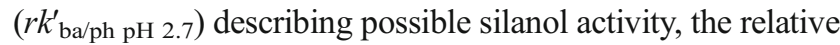
retention factor of triphenylene/o-terphenyl ( $\left.r k_{\text {tri/o-ter }}^{\prime}\right)$ giving an indication of steric selectivity, and the retention factor of 2,2'-dipyridyl $\left(k_{2,2^{\prime}-\mathrm{d}}^{\prime}\right)$ estimating silanol activity and metal impurities $[19,20]$. After choosing a specific reference column or selecting four reference parameters corresponding to the selected reference phase, the $F_{\mathrm{KUL}}$ value for column $i$, being the sum of the squares of the differences between each parameter value of the reference stationary phase and that of column $i$ is calculated:

$$
\begin{aligned}
& F_{\mathrm{KUL}}=\left(k_{a m b, r e f}-k_{a m b, i}\right)^{2}
\end{aligned}
$$

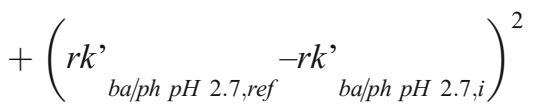

$$
\begin{aligned}
& +\left(\begin{array}{cc}
r k^{\prime} & -r k{ }^{\prime} \\
&
\end{array}\right)^{2}
\end{aligned}
$$

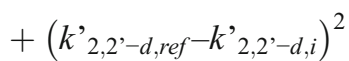

In order to guarantee that the parameters are ascribed the same weight, each of them is auto-scaled ("normalized") before its introduction in Eq. (1) [21]. 
Next, a comparison is conducted between various brands of stationary phases of column $i$ and the reference phases. The higher similarity of column $i$ to the reference column, the lower the $F_{\mathrm{KUL}}$ value was observed and column $i$ goes higher in the ranking list (high-ranked columns). Higher $F_{\mathrm{KUL}}$ values of column $i$ confirm the existence of significant dissimilarities between them, and this puts the stationary phase lower on the ranking list arranged by the increasing $F_{\text {KUL }}$ value. This convenient and useful simplification, combining four different contributors into a single parameter, has caused that all calculated $F_{\mathrm{KUL}}$ values for the tested stationary phases are related to a single reference phase. The stationary phases with $F_{\mathrm{KUL}}<2$ are commonly considered high ranking, and offering the highest probability of finding an appropriate alternative. The columns with $2<F_{\mathrm{KUL}}<6$ are deemed average, whereas columns with $F_{\mathrm{KUL}}>6$ are treated as low ranking, offering the lowest probability of selecting the appropriate stationary phase.

Literature presents a few reports focused on the correlation between the KUL approach versus other column classification methods [22-25] and numerous reports confirming usefulness of the KUL test procedure for real pharmaceutical applications $[18,20-26]$. On the other hand, most of those comparative studies were based on correlating the KUL ranking list only with real selectivity in pharmaceutical analysis described by the European Pharmacopoeia (Ph. Eur.) system suitability test (SST) values or the chromatographic response function indicating the overall selectivity $[18,20-22,26]$. Only few papers have reported more detailed correlation between the KUL test results to classify stationary phases and the column test performance in real pharmaceutical analyses. These comparative studies were based on experimental data including the retention parameters of alfuzosin and its two impurities [27] or caffeine and its four related compounds [28]. In both column test performances, the analytes were eluted in isocratic conditions, similarly to its use in the KUL. Thus, it seems to be interesting whether the KUL method can be considered as a useful tool to classify the stationary phases when gradient elution of the mobile phase is required for simultaneous LC analysis of many analytes.

Therefore, the column classification system based on the KUL procedure was compared against selectivity of the separation of lamotrigine and its seven related compounds (Fig. 1), using a set of 28 stationary phases previously tested under the KUL conditions. The LC analysis was carried out according to the Ph. Eur. monograph [29], where gradient elution of the compounds of interest was prescribed. Moreover, the SST requires that peak-to-valley ratio $(p / v)$ between height above the baseline of the peak due to impurity $\mathrm{G}$ and height above the baseline of the lowest point of the curve separating this peak from the peak due to lamotrigine a<smiles>Nc1nnc(-c2cccc(Cl)c2Cl)c(N)n1</smiles>

C<smiles>N#C/C(=N/N=C(N)N)c1cccc(Cl)c1Cl</smiles>

e<smiles>O=c1[nH]nc(-c2cccc(Cl)c2Cl)c(=O)[nH]1</smiles><smiles>Nc1nc(NC(=O)c2cccc(Cl)c2Cl)nnc1-c1cccc(Cl)c1Cl</smiles>

h<smiles>Nc1nnc(-c2ccc(Cl)cc2Cl)c(N)n1</smiles>

Fig. 1 Chemical structure of a lamotrigine, b impurity A, c impurity B, d impurity C, e impurity D, f impurity E, g impurity F, and h impurity G

was minimum 1.2. Therefore, the SST test was established for all RP-LC columns studied. Next, upon selection of a Hypersil BDS 100-C18 $150 \times 4.6 \mathrm{~mm}, 5 \mu \mathrm{m}$ (HYB) column as reference, the KUL ranging list was calculated and confronted with the column selectivity towards the tested analytes described by the overall criterion (the $p / v$ ratios). Next, the retention parameters of lamotrigine and its seven related substances were calculated for all stationary phases, including the retention time $\left(t_{\mathrm{R}}\right)$ and the resolution of the peaks of interest $\left(R_{\mathrm{S}}\right)$, providing a detailed description of the column performance in real analysis. Finally, a chemometric method, namely factor analysis (FA), was used for checking whether the column classes closely related in terms of their KUL characteristics showed similar separation for lamotrigine and its related compounds. For a more detailed interpretation of the obtained theoretical results of KUL method and their column performance in real separation, the same numbers of 1-28 were assigned for the stationary phases in both data sets. Moreover, the localizations of individual stationary phases in column classes were correlated with the SST parameters. Additionally, the second $\mathrm{Ph}$. Eur. requirement involves the $t_{\mathrm{R}}$ of about $7 \mathrm{~min}$ for lamotrigine and the relative retention times $\left(t_{\mathrm{RR}}\right)$ of about 1.1, 1.3, 1.7, and 1.8 for impurities G, A, E, and F, respectively. These parameters were also taken into account when the application of the KUL method in 
the selectivity study involving lamotrigine and its related impurities was examined.

\section{Material and methods}

\section{Column examination}

Twenty-eight RP-LC stationary phases examined in this study were donated by the manufacturers or the distributors. Their specifications are reported in Table 1.
KUL method

\section{Materials}

Amylbenzene, benzylamine, 2,2'-dipyridyl, o-terphenyl, triphenylene, and uracil were supplied by Sigma-Aldrich (St. Louis, MO, USA) whereas phenol was purchased from POCH (Gliwice, Poland). Methanol and potassium dihydrogen phosphate of HPLC grade purchased from J.T. Baker (Deventer, Netherlands) while orthophosphoric acid was delivered by Merck (Darmstadt, Germany). All chemicals

Table 1 List of C18 RP-LC tested stationary phases and their specifications as provided by the manufacturer

\begin{tabular}{|c|c|c|c|c|c|c|c|c|}
\hline $\begin{array}{l}\text { Number of the } \\
\text { column }\end{array}$ & Name of the column & $\begin{array}{l}\text { Manufacturer/ } \\
\text { supplier }\end{array}$ & $\begin{array}{l}\text { Carbon } \\
\text { load [\%] }\end{array}$ & $\begin{array}{l}\text { Pore size } \\
{[\AA]}\end{array}$ & $\begin{array}{l}\text { Surface Area } \\
{\left[\mathrm{m}^{2} / \mathrm{g}\right]}\end{array}$ & Silica & Endcap. & Abbreviation \\
\hline 1 & Hypersil BDS C18 & Thermo & 11 & 130 & 170 & A & + & HYB \\
\hline 2 & Hypersil Elite C18 & Thermo & 15 & 114 & 250 & A & + & HYE \\
\hline 3 & Xbridge C18 & Waters & 18 & 130 & 185 & $\mathrm{~B}$ & + & $\mathrm{XBC} 18$ \\
\hline 4 & Discovery C18 & Supelco & 12 & 180 & 200 & $\mathrm{~B}$ & + & DISC \\
\hline 5 & ACE 5 C18 & $\mathrm{ACT}$ & 15.5 & 100 & 300 & $\mathrm{~B}$ & + & AC18 \\
\hline 6 & ACE 5 C18-AR & $\mathrm{ACT}$ & 15.5 & 100 & 300 & $\mathrm{~B}$ & + & AAR \\
\hline 7 & Inertsil ODS2 & GL Science & 18.5 & 150 & 320 & $\mathrm{~B}$ & + & ING \\
\hline 8 & $\begin{array}{l}\text { Nucleosil 100-5 C18 } \\
\text { HD }\end{array}$ & $\begin{array}{l}\text { Macherey- } \\
\text { Nagel }\end{array}$ & 20 & 100 & 350 & A & + & NUH \\
\hline 9 & ACE 5 AQ & $\mathrm{ACT}$ & 14 & 100 & 300 & $\mathrm{EP}$ & + & AAQ \\
\hline 10 & Wakosil II 5 C18 HG & SGE & 15 & 120 & 300 & B & + & WAH \\
\hline 11 & $\begin{array}{l}\text { Nucleosil 100-5 C18 } \\
\text { Nautilus }\end{array}$ & $\begin{array}{l}\text { Macherey- } \\
\text { Nagel }\end{array}$ & 16 & 100 & 350 & $\mathrm{EP}$ & + & NUN \\
\hline 12 & Inertsil ODS2 & Hichrom & 18.5 & 150 & 320 & $\mathrm{~B}$ & + & INH \\
\hline 13 & YMC Pack ODS-AQ & YMC & 14.1 & 120 & 300 & $\mathrm{~B}$ & + & YPAQ \\
\hline 14 & $\begin{array}{l}\text { Nucleosil 100-5 C18 } \\
\text { AB }\end{array}$ & $\begin{array}{l}\text { Macherey- } \\
\text { Nagel }\end{array}$ & 24 & 100 & 350 & A & + & NUA \\
\hline 15 & Aqua $\mathrm{C} 18$ & Phenomenex & 15 & 125 & 320 & B & + & AQ18 \\
\hline 16 & Kromasil 100-5 C18 & Akzo Nobel & 19 & 100 & 340 & $\mathrm{~B}$ & + & KRO \\
\hline 17 & $\begin{array}{l}\text { Nucleodur C18 } \\
\text { Pyramid }\end{array}$ & $\begin{array}{l}\text { Macherey- } \\
\text { Nagel }\end{array}$ & 14 & 110 & 340 & $\mathrm{~B}$ & + & NUP \\
\hline 18 & ACE 5 C18-HL & $\mathrm{ACT}$ & 20 & 90 & 400 & $\mathrm{~B}$ & + & AHL \\
\hline 19 & Nucleosil 100-5 C18 & $\begin{array}{l}\text { Macherey- } \\
\text { Nagel }\end{array}$ & 15 & 100 & 350 & A & + & NU18 \\
\hline 20 & Prodigy ODS3 & Phenomenex & 15.5 & 100 & 450 & B & + & PODS3 \\
\hline 21 & SunFire C18 & Waters & 16 & 100 & 340 & $\mathrm{~B}$ & + & SUN \\
\hline 22 & Luna C18 & Phenomenex & 17.5 & 100 & 400 & $\mathrm{~B}$ & + & LUN \\
\hline 23 & Aquasil C18 & Thermo & 12 & 100 & 310 & $\mathrm{EP}$ & + & AQSIL \\
\hline 24 & Zorbax SB-Aq & Agilent & proprietary & 80 & 180 & EP & - & ZSBA \\
\hline 25 & Xbridge Shield RP18 & Waters & 17 & 130 & 185 & $\mathrm{EP}$ & + & $\mathrm{XBSH}$ \\
\hline 26 & $\begin{array}{l}\text { Symmetry Shield } \\
\text { RP18 }\end{array}$ & Waters & 17 & 100 & 335 & $\mathrm{EP}$ & + & SYSH \\
\hline 27 & Spherisorb ODS1 & Waters & 6.2 & 80 & 220 & A & - & SODS1 \\
\hline 28 & Spherisorb ODS2 & Waters & 11.5 & 80 & 220 & A & + & SODS2 \\
\hline
\end{tabular}

Each column has a length of $150 \mathrm{~mm}$, a diameter of $4.6 \mathrm{~mm}$, and particle size of $5 \mathrm{um}$

$A$ "traditional," acidic silica gel, $B$ "high purity," more neutral silica gel, EP embedded or end-capped polar group 
were used as received without further purification. Water was pretreated in a Milli-Q Water Purification System (Millipore Corporation, Bedford, MA, USA).

\section{Equipment and LC conditions}

All LC separations were carried out using a Waters system (Milford, MA, USA) consisting of 2,695 Separation Module, Column Heater/Cooler with three-column selector valve (Rheodyne RV500-100), 2,996 Photodiode Array Detector and the Empower 2 software for data acquisition. In each method, the column temperature was maintained at $40{ }^{\circ} \mathrm{C}$, and the analytes were monitored at $254 \mathrm{~nm}$. The flow rate was $1 \mathrm{~mL} / \mathrm{min}$, and the injected sample volume was $20 \mu \mathrm{L}$.

\section{Column characterization}

In order to calculate the relative retention factor of

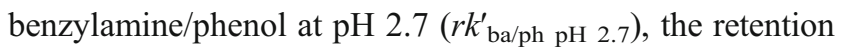

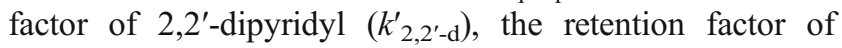
amylbenzene $\left(k_{\mathrm{amb}}^{\prime}\right)$, and the relative retention factor of triphenylene/o-terphenyl $\left(r k_{\text {tri/o-ter }}^{\prime}\right)$, three isocratic chromatographic methods in a defined order (A-B-C) were used as previously reported [22]. In these calculations, the dead volume obtained with uracil in method $\mathrm{C}$ was used. All measurements were repeated three times, and the RSD values were lower than $1 \%$. Next, four column parameter values for all examined stationary phases were calculated. Finally, upon selection of the HYB column as reference, the $F_{\mathrm{KUL}}$ values for the other stationary phases were calculated according to Eq. 1 using the software available online at http:// pharm.kuleuven.be/pharmchem/Pages/ccs.html. These data are summarized in Table 2.

Column performance for the determination of lamotrigine

\section{Materials}

In addition to the reagents from Section "KUL method: Materials," lamotrigine for system suitability CRS composed of lamotrigine (6-(2,3-dichlorophenyl)-1,2,4-triazine-3,5-diamine) and the impurity G (6-(2,4-dichlorophenyl)-1,2,4trazine-3,5-diamine) were obtained from EDQM (Strasbourg, France). Lamotrigine $(100.0 \%)$, the impurity A (3-amino6-(2,3-dichlorophenyl)-1,2,4-triazin-5(4H)-one) (99.7\%), the impurity B ((2E)-[2-(diaminomethylidene)diazanylideno](2,3dichlorophenyl)acetonitrile) $(99.1 \%)$, the impurity C ((2Z)[2-(diaminomethylidene)diazanylideno](2,3-dichlorophenyl)acetonitrile) (99.4\%), the impurity D (6-(2,3-dichlorophenyl)1,2,4-trazine-3,5(2H,4H)-dione) ( $98.8 \%)$, and the impurity $\mathrm{F}$ (N-[5-amino-6-(2,3-dichlorophenyl)-1,2-4-triazin-3-yl]-2,3dichlorobenzamide) $(94.6 \%)$ were produced by Polpharma
SA (Starogard Gdański, Poland). The impurity E (2,3dichlorobenzoic acid) was supplied by Fluka-Sigma-Aldrich (St. Louis, MO, USA). All solvents and reagents were of Ph. Eur. quality. The stock standard solution of lamotrigine and the impurity $\mathrm{G}$ was prepared by dissolution of $5.0 \mathrm{mg}$ of lamotrigine for system suitability CRS in $2.5 \mathrm{~mL}$ of methanol and diluted to $50 \mathrm{~mL}$ with a $10.3 \mathrm{~g} / \mathrm{L}$ solution of hydrochloric acid. Next, $1.0 \mathrm{~mL}$ of the stock test solution was diluted to $10.0 \mathrm{~mL}$ with a $10.3 \mathrm{~g} / \mathrm{L}$ solution of hydrochloric acid resulting in lamotrigine concentration of $0.01 \mathrm{mg} / \mathrm{mL}$ in the presence of impurity $\mathrm{G}$ at the concentration of $50 \mathrm{ng} / \mathrm{mL}$. Finally, this reference solution was injected into the HPLC system to check compliance with the SST requirement. The stock standard solution of the impurities A-F was prepared by dissolution of $10.0 \mathrm{mg}$ of each impurity in $100 \mathrm{~mL}$ of methanol. Next, for preparing of the working standard solution of the impurities A-F, $1.0 \mathrm{~mL}$ of this stock solution was diluted to $10 \mathrm{~mL}$ with a $10.3 \mathrm{~g} / \mathrm{L}$ solution of hydrochloric acid. The working standard solution of lamotrigine in the presence of the impurities $A-G$ was prepared by dissolution of $8.0 \mathrm{mg}$ of lamotrigine in $2.5 \mathrm{~mL}$ of methanol, addition of $20.0 \mathrm{~mL}$ of stock standard solution of lamotrigine and the impurity $G$ prepared from lamotrigine for system suitability CRS, addition of $1.0 \mathrm{~mL}$ of working standard solution of the impurities A-F, and dilution to $50 \mathrm{~mL}$ with a $10.3 \mathrm{~g} / \mathrm{L}$ solution of hydrochloric acid. Consequently, the working standard solution of lamotrigine at the concentration of $0.2 \mathrm{mg} / \mathrm{mL}$ and in the presence of its seven impurities, each at the level of $0.1 \%$ of the parent substance, has been finally analyzed. The HPLC grades of acetonitrile, triethylamine, and hydrochloric acid used for preparing of the mobile phase and the standard solutions of the tested analytes were purchased from J.T. Baker (Deventer, Netherlands).

\section{Equipment and LC conditions}

The same LC equipment was used as that mentioned in Section "KUL method: Equipment and LC conditions." According to the $\mathrm{Ph}$. Eur. monograph, the separation of lamotrigine and its related compounds was performed with gradient elution of solvent A (mix 1 volume of triethylamine and 150 volumes of a $2.7 \mathrm{~g} / \mathrm{L}$ solution of potassium dihydrogen phosphate adjusted to $\mathrm{pH} 2.0$ with orthophosphoric acid) and solvent B (acetonitrile). During the analysis, the following gradient program of the mobile phase was realized: (1) 0-4 min, $85 \% \mathrm{~A}, 15 \% \mathrm{~B}$; (2) 4-14 min, gradient elution formed from 15 to $80 \% \mathrm{~B}$; (3) 14-14.5 min, from 80 to $15 \% \mathrm{~B}$; and (4) $14.5-25 \mathrm{~min}, 15 \% \mathrm{~B}$ to achieve column equilibration. The flow rate was maintained at $1 \mathrm{~mL} / \mathrm{min}$, and the UV detection was performed at $270 \mathrm{~nm}$. The LC system was operated at $35{ }^{\circ} \mathrm{C}$. The injected sample volume was $10 \mu \mathrm{L}$. 


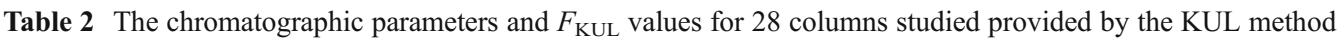

\begin{tabular}{|c|c|c|c|c|c|c|}
\hline \multirow[t]{2}{*}{ Analytical column } & \multicolumn{4}{|c|}{ Column parameters } & \multirow[t]{2}{*}{$F$} & \multirow{2}{*}{$\begin{array}{l}\text { The position in } \\
\text { the ranking list } \\
\text { (column no.) }\end{array}$} \\
\hline & $k_{\mathrm{amb}}^{\prime}$ & $r k_{\text {tri/o-ter }}^{\prime}$ & $r k_{\mathrm{ba} / \mathrm{ph} \mathrm{pH} 2.7}^{\prime}$ & $k_{2,2^{\prime}-\mathrm{d}}^{\prime}$ & & \\
\hline HYB & 3.480 & 1.569 & 0.136 & 5.123 & 0 & 1 \\
\hline HYE & 4.450 & 1.502 & 0.121 & 6.807 & 0.407 & 2 \\
\hline $\mathrm{XBC} 18$ & 3.361 & 1.392 & 0.128 & 5.518 & 0.425 & 3 \\
\hline DISC & 2.780 & 1.447 & 0.087 & 4.408 & 0.473 & 4 \\
\hline $\mathrm{AC} 18$ & 4.489 & 1.505 & 0.097 & 6.833 & 0.498 & 5 \\
\hline AAR & 3.483 & 1.698 & 0.099 & 8.180 & 0.587 & 6 \\
\hline ING & 5.170 & 1.445 & 0.051 & 8.667 & 0.871 & 7 \\
\hline NUH & 5.099 & 1.482 & 0.093 & 8.049 & 1.157 & 8 \\
\hline AAQ & 2.232 & 1.322 & 0.077 & 7.271 & 1.548 & 9 \\
\hline WAH & 4.913 & 1.353 & 0.070 & 7.422 & 1.567 & 10 \\
\hline NUN & 2.734 & 1.827 & 0.023 & 5.828 & 1.744 & 11 \\
\hline INH & 4.257 & 1.639 & 0.062 & 8.431 & 1.749 & 12 \\
\hline$Y P A Q$ & 4.164 & 1.258 & 0.073 & 9.137 & 2.124 & 13 \\
\hline$N U A$ & 3.658 & 1.964 & 0.097 & 6.021 & 2.157 & 14 \\
\hline$A Q 18$ & 4.932 & 1.277 & 0.096 & 9.243 & 2.292 & 15 \\
\hline KRO & 6.199 & 1.491 & 0.091 & 9.038 & 2.599 & 16 \\
\hline NUP & 4.682 & 1.259 & 0.060 & 9.879 & 2.674 & 17 \\
\hline AHL & 6.369 & 1.535 & 0.087 & 8.950 & 2.786 & 18 \\
\hline NU18 & 3.360 & 1.634 & 0.115 & 14.430 & 2.794 & 19 \\
\hline PODS3 & 5.476 & 1.246 & 0.078 & 8.416 & 2.933 & 20 \\
\hline SUN & 5.479 & 1.231 & 0.038 & 9.124 & 3.58 & 21 \\
\hline LUN & 5.509 & 1.172 & 0.050 & 9.012 & 4.036 & 22 \\
\hline AQSIL & 2.971 & 1.825 & 0.163 & 15.163 & 4.124 & 23 \\
\hline$Z S B A$ & 0.863 & 1.192 & 0.109 & 9.989 & 4.437 & 24 \\
\hline XBSH & 2.296 & 2.111 & 0.046 & 4.456 & 4.684 & 25 \\
\hline SYSH & 3.771 & 2.212 & 0.022 & 6.575 & 6.25 & 26 \\
\hline SODS2 & 4.044 & 1.657 & 0.366 & 17.275 & 7.809 & 27 \\
\hline SODS1 & 2.041 & 1.865 & 0.334 & 23.641 & 14.641 & 28 \\
\hline
\end{tabular}

Meaning of symbols is explained in the text. The columns non-suitable for the LC analysis of lamotrigine according to the SST test are indicated in bold. The stationary phases non-suitable for the separation of the analytes according to the second Ph. Eur. recommendation are indicated in italics

\section{Column test performance}

Firstly, the column test performance based on the LC separation of the samples containing lamotrigine for system suitability CRS which had been prepared as described in Section "Column performance for the determination of lamotrigine: Materials," and analyzed in the chromatographic conditions presented in Section "Equipment and LC condition," was carried out on HYB column. Next, the same LC analysis was performed using other columns examined. All measurements were repeated three times. Then, the peak-to-valley $(p / v)$ ratios were evaluated in accordance to the SST as described in the Ph. Eur. monograph, and these data are presented in Table 3. Further, the LC analysis of a sample containing lamotrigine and the seven impurities (A, B, C, D, E, F, and G) was conducted ( $n=3)$. The representative chromatograms of the sample containing the compounds of interest obtained on HYB, HYE, and SODS1 are shown in Fig. 2a-c, respectively. Then, the $t_{\mathrm{R}}$ of the analytes and the $R_{\mathrm{S}}$ of peaks of interest which were calculated from preceding peak obtained during LC separation of lamotrigine in the presence of its impurities were established for all stationary phases. Moreover, the relative retention times $\left(t_{\mathrm{RR}}\right)$ of the analytes expressed as the percentage of the $t_{\mathrm{RR}}$ values recommended by the Ph. Eur. monograph $\left(\% t_{\mathrm{RR}}\right)$ were calculated. These experimental data describing in detail each real pharmaceutical analysis are summarized in Table 3.

Data treatment

The theoretical KUL results for the 28 columns examined and their application in pharmaceutical practice for separation of lamotrigine along with their impurities were subject to a 


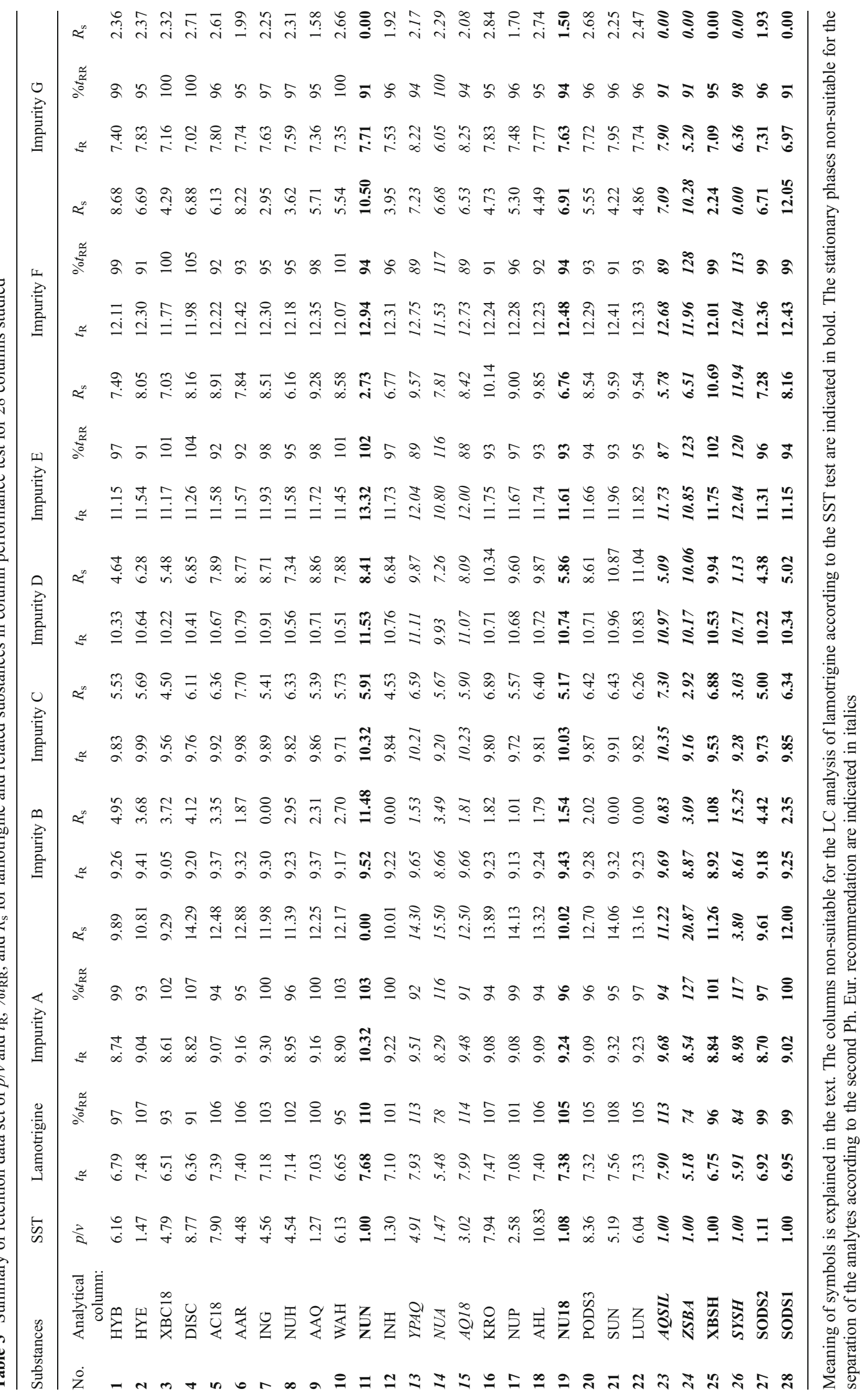


Fig. 2 LC analysis of the sample containing lamotrigine and impurities $\mathrm{A}-\mathrm{G}$ at the concentration levels of $0.2 \mathrm{mg} \mathrm{mL}^{-1}$ for parent substance and $0.1 \%$ for the related compounds performed on a HYP, b HYE, and c SODS1 column, respectively a

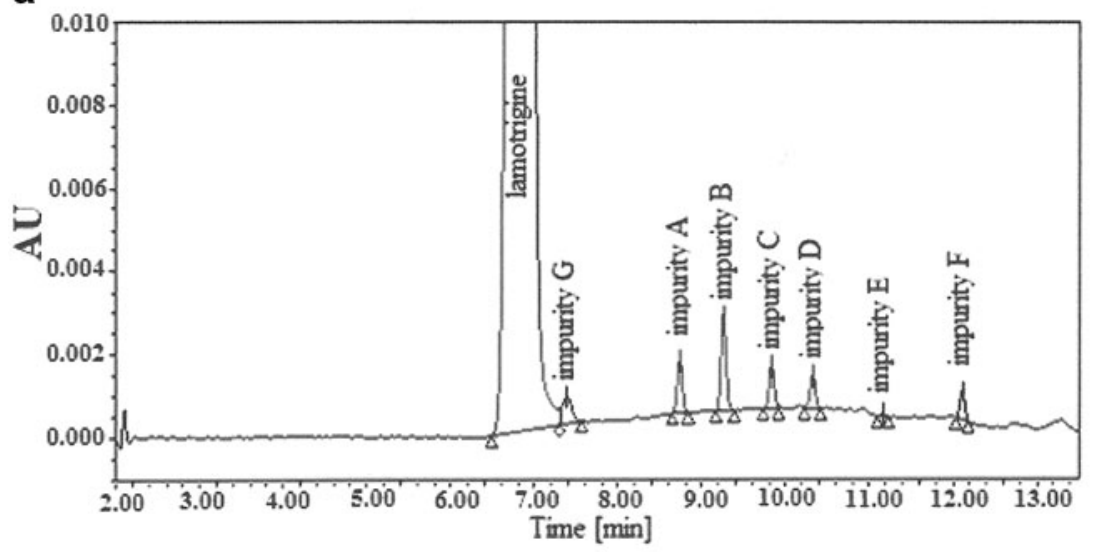

b

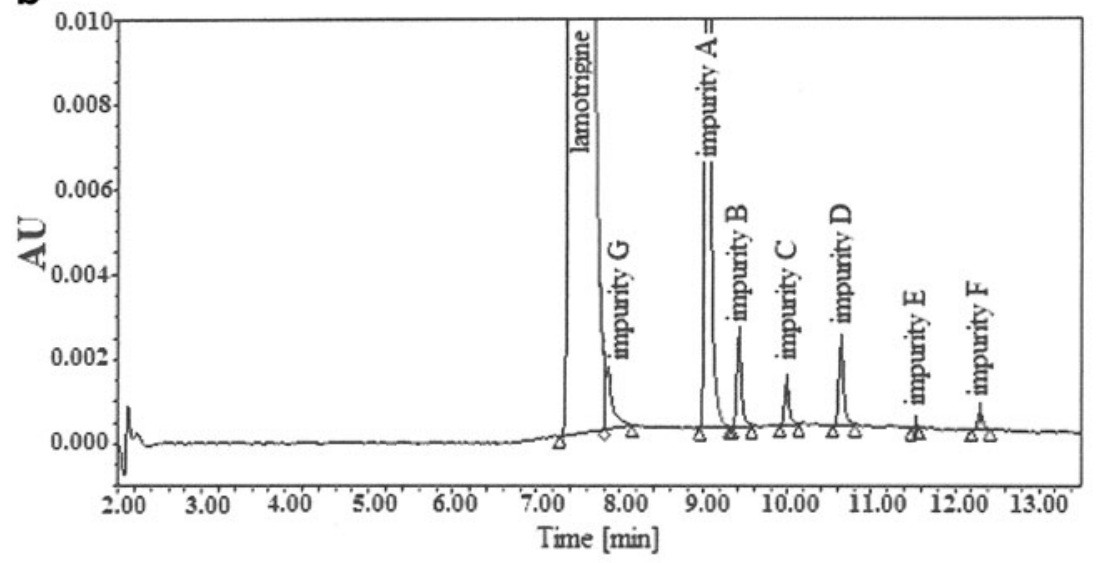

C

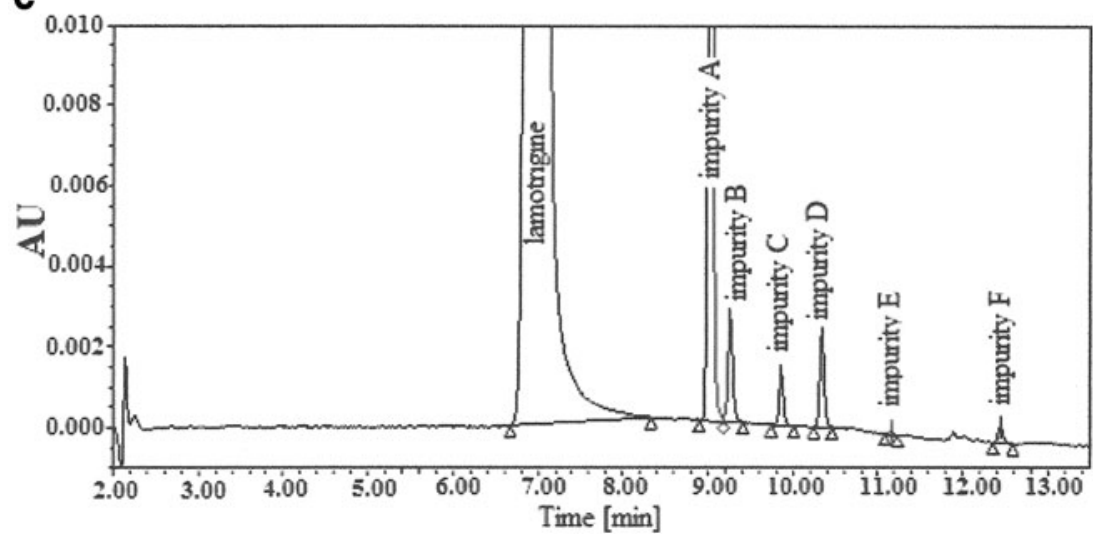

comparative study in order to check whether the KUL test procedure could be used to facilitate RP-LC column selection in the considered pharmaceutical analysis. To visualize the relationships between the data sets containing many variables and objects, a chemometric technique, namely FA based on the algorithm without rotation mode was applied using the Statistica 9.0 package (StatSoft, Tulsa, USA). The stationary phase 1-28 numbering in accordance with the $F_{\mathrm{KUL}}$ values provided by the KUL method (Table 2) was retained unchanged in these calculations. Firstly, FA extracted from the standardized four chromatographic parameters provided by the KUL method, was calculated for all stationary phases. The obtained FA plots projecting the variables and the objects (columns) in two-dimensional space are shown in Fig. 3a and b, respectively. Then, FA based on the standardized retention parameters of $t_{\mathrm{R}}$ and $R_{\mathrm{S}}$ for lamotrigine and its seven impurities (A, B, C, D, E, F, and $\mathrm{G}$ ) as well as the $p / v$ values established according to the SST test (Table 3) for all stationary phases was performed. Two-dimensional plots for the variables and 
Fig. 3 Projection of the variables (a) and objects (b) onto the space of the first two PFs (PF1 and PF2) extracted from FA four column parameters provided by KUL test procedure for the 28 stationary phases studied a

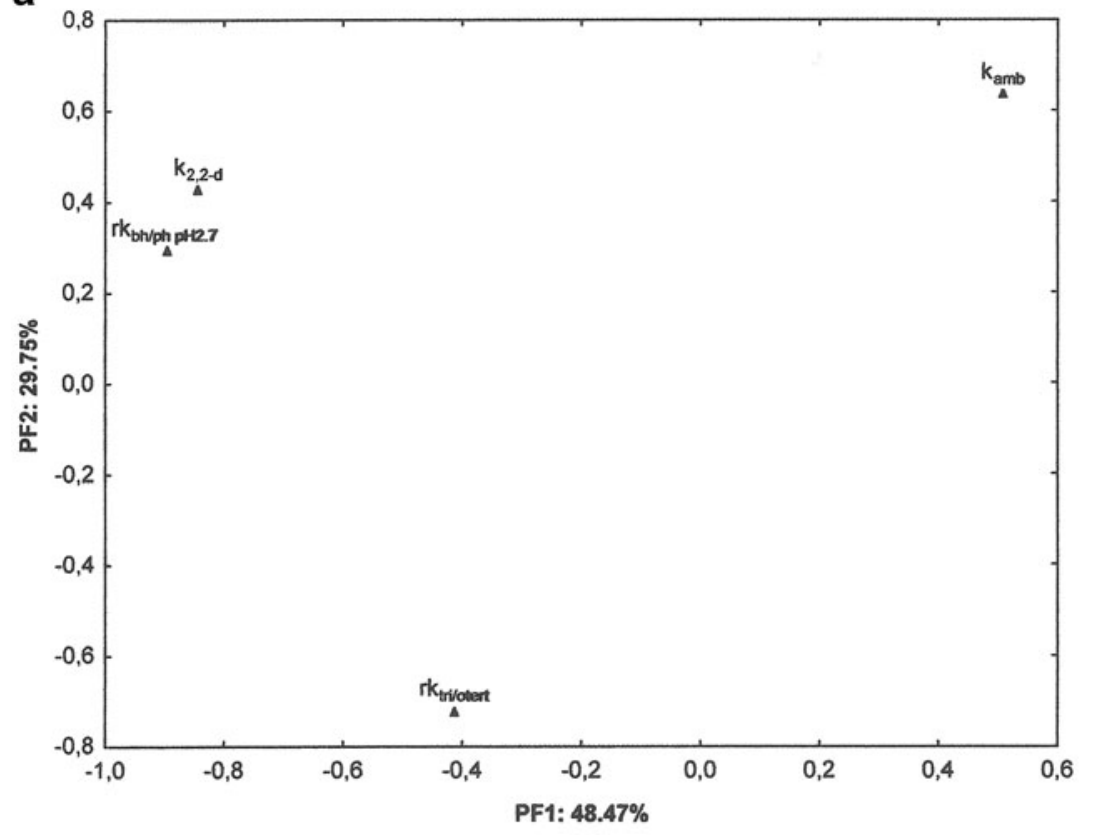

b

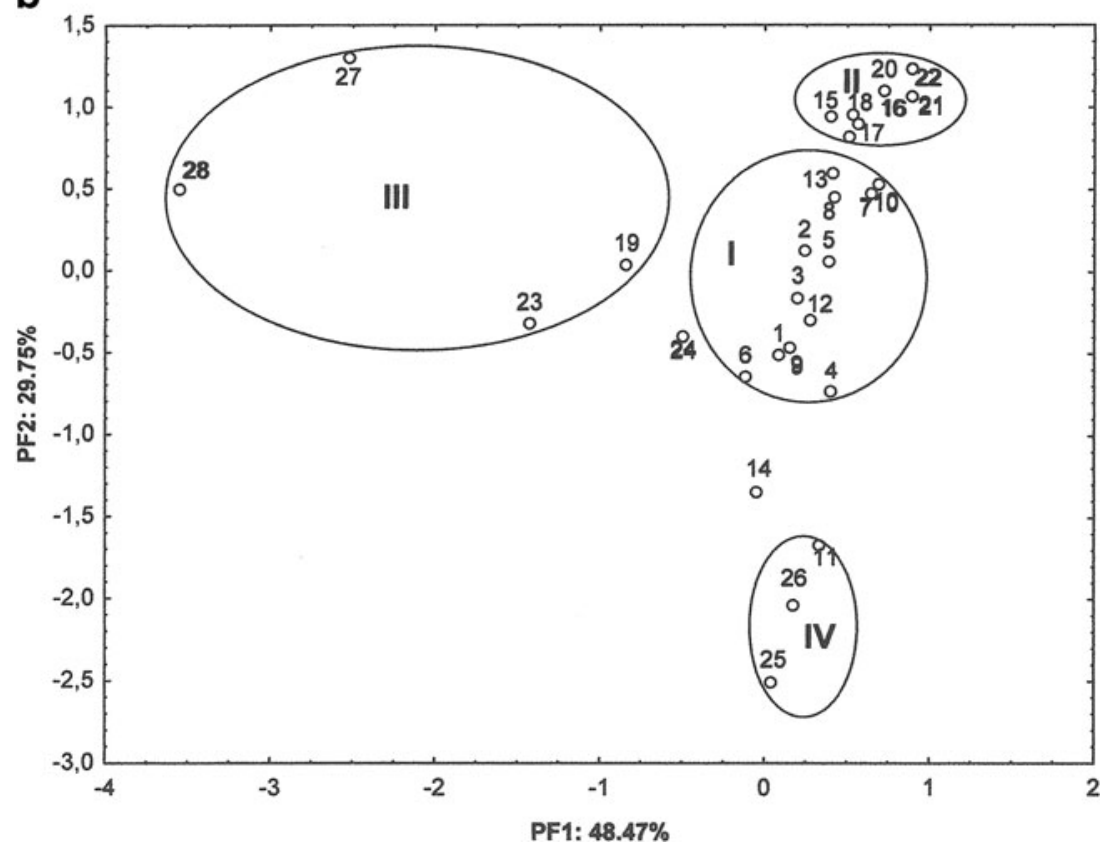

the columns resulting from the FA are illustrated in Fig. $4 \mathrm{a}$ and $\mathrm{b}$, respectively.

\section{Results and discussion}

\section{Column characterization}

The KUL approach involves using selected test solutes which are analyzed in strictly set LC conditions. Thus, each column in the KUL ranking system is characterized using sets of four column parameters, which enables establishment of the $F_{\mathrm{KUL}}$ values. The column parameter values established for 28 stationary phases are summarized in Table 2 . In accordance with the

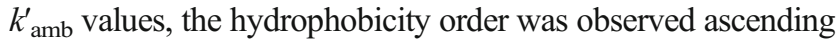
from ZSBA to AHL. Silanol activity described by $r k^{\prime}{ }_{\mathrm{ba} / \mathrm{ph} \text { pH2.7 }}$ is due to the silanol groups, which remain on the surface because of incomplete end-capping. Their presence plays an important role in the analyte retention mechanism. The highest values of the parameter were calculated for SODS2 and SODS1, while the lowest were found for SYSH and NUN. The values of $r k_{\text {tri/o-ter }}^{\prime}$ and hence, the steric selectivity of the 
Fig. 4 Two-dimensional FA plots of the variables (a) and objects (b) based on the retention parameters $\left(t_{\mathrm{R}}\right.$ and $\left.R_{\mathrm{S}}\right)$ of the analytes during column performance for the LC analysis of lamotrigine and its related substances

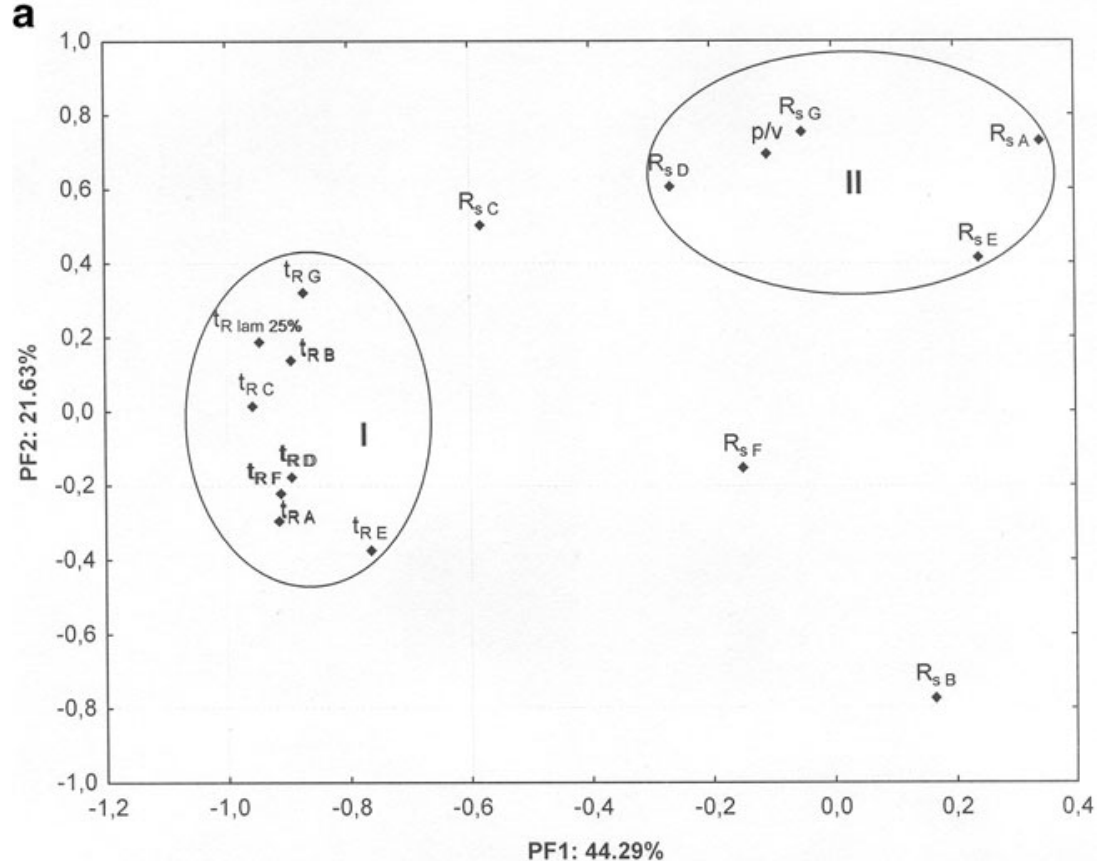

b

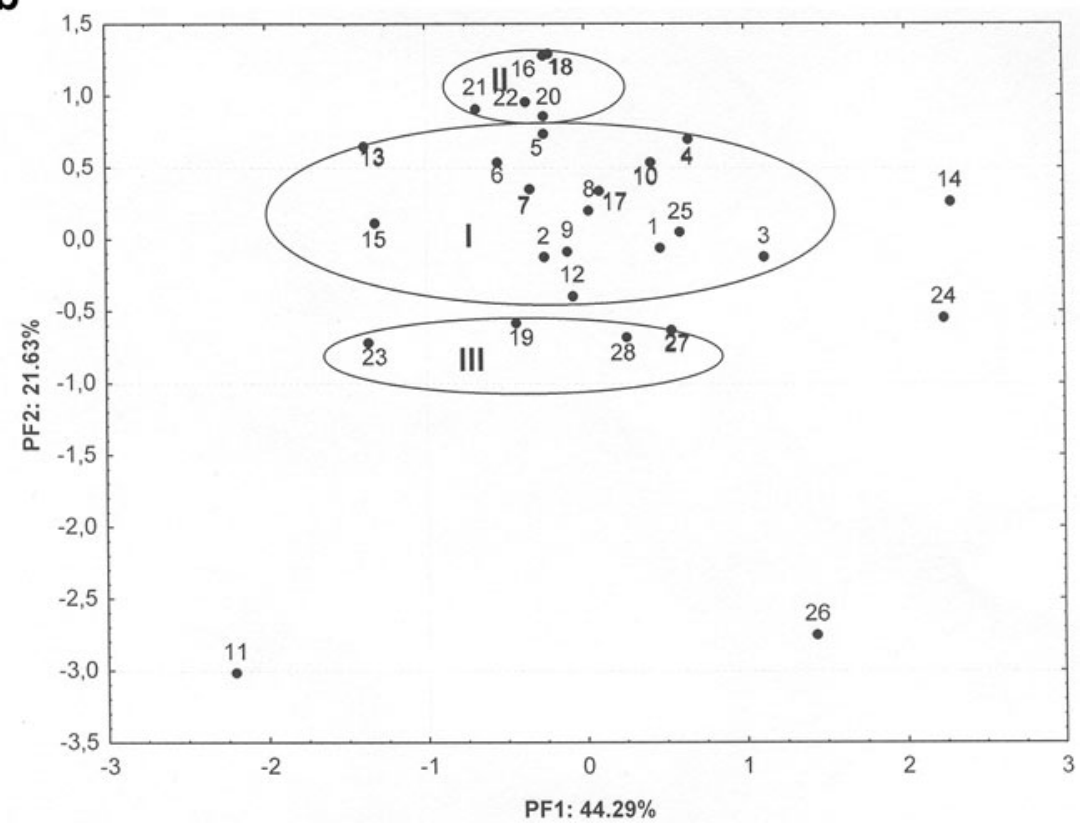

stationary phase were observed to rise beginning with LUN and ending with SYSH. Metal contamination may enhance silanol acidity polarity and the chelate formation potential. Based on the numerical data from Table 2, the highest value of the $k_{2,2^{\prime} \mathrm{d}}$ parameter indicating silanol activity and metal impurities were calculated for SODS1, whereas the lowest was obtained for DISC. In the study, the reference stationary phase (HYB column) was characterized as having a low hydrophobic nature, an intermediate potential for steric selectivity, and revealing lower silanol activity and metal contamination despite the fact that type-A silica was used (Table 1). When KUL ranking list was taken into account, 11 columns were found to be high-ranked $\left(F_{\mathrm{KUL}}<2\right)$, where HYE also based on the type-A silica with lower $k_{2,2^{\prime} \mathrm{d}}^{\prime}$ parameter was considered as most similar to the reference column $\left(F_{\mathrm{KUL}}=0.407\right)$. Thirteen stationary phases were identified as intermediate, while columns SYSH to SODS1 were described as low-ranked ones $\left(F_{\mathrm{KUL}}>6\right)$, which indicates that their chromatographic properties were significantly different from the reference column. They were characterized by higher $r k_{\text {tri/o-ter }}^{\prime}$ parameters.

Next, for clearer interpretation of the theoretical KUL results for the 28 columns studied, FA based on the 
standardized four chromatographic parameters was performed. The two-dimensional plots for the variables and objects derived from KUL data set (Table 2) are shown in Figs. $3 \mathrm{a}$ and $4 \mathrm{~b}$, respectively. The use of chemometric techniques for the evaluation of the similarities and dissimilarities of the RP-LC stationary phases has been reported [5, 9, 15, 23, 30-33], but only few papers have presented the possibility of the multivariate methods for the column selectivity study in detail so far $[27,28$, 33]. It can be highlighted that the variability of $r k_{\mathrm{ba} / \mathrm{ph} \mathrm{pH} 2.7}^{\prime}$ and $k_{2,2^{\prime} \mathrm{d}}^{\prime}$ was mainly explained by the first principal factor (PF1). These variables positioned themselves close to each other on the left on the upper side of the plot (Fig. 3a). The localization of the variables and objects on the PF2 axes mainly resulted from the variability of $r k_{\text {tri/o-ter }}^{\prime}$ and $k_{\text {amb. These variables were found outliers }}^{\prime}$ on the bottom part and on the upper side of the graph, respectively. In summary, $78.22 \%$ of the variance of the analyzed data set can be explained by the first two PFs. Columns with high-ranked positions $\left(F_{\mathrm{KUL}}<2\right)$ (nos. $1-10,12)$ as well as the stationary phase no. 13 $\left(F_{\mathrm{KUL}}=2.124\right)$ were found in cluster I located on the right in the middle part of the graph (Fig. 3b). Their characteristics include lower and intermediate $k_{2,2^{\prime} \mathrm{d}}$ and $r k_{\text {tri/o-ter }}^{\prime}$ parameters (Table 2). For them, except of nos. $1,2,8$, and 9 , the type-B silica was used. The columns positioned as intermediate in the KUL ranking list (nos. 15-18, 20-22), identified by higher $k_{\text {amb }}^{\prime}$ against lower

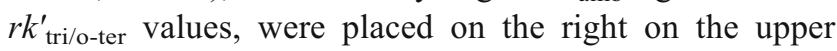
side of the FA plot. These stationary phases were based on the type-B silica but the surface areas calculated for them were higher than observed in cluster I (Table 1). On the other hand, low-ranked stationary phases with the type-A silica (nos. 27 and 28) were found together with column nos. 19 and 23 within cluster III located on the left on the upper part of the plot (Fig. 3b). For them, high silanol activity probably related with high level of the metal contaminant which leads to signifi-

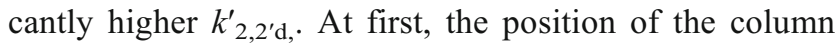
no. 11 seems to be surprising because this column was positioned together with the stationary phase nos. 25 and 26 within cluster IV falling in the bottom side of the graph. On the other hand, each of these stationary phases has EP group resulting in lowest values of $r k_{\mathrm{bh} / \mathrm{ph}}^{\prime} \mathrm{pH} 2.7$ parameters, while $r k_{\text {tri/o-ter }}^{\prime}$ values were the highest. Only column no. 14 was itself near column no. 24 between clusters I, III, and IV. For these station-

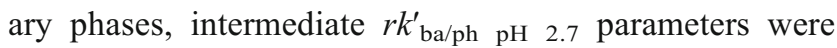
calculated by the KUL test procedure. These columns have different type of the silica (A and EP), but their common feature was probably high carbon load. Unfortunately, this parameter was proprietary for stationary phase no. 24 .
Comparison based on the overall criterion of column selectivity in separation of lamotrigine

A test of the reliability of KUL column classification system in pharmaceutical practice was based on LC separation of lamotrigine and its impurities, which was performed with gradient elution according to the $\mathrm{Ph}$. Eur. monograph [29], using set of 28 new RP-LC C18 stationary phases. For this $\mathrm{LC}$ analysis, the $\mathrm{Ph}$. Eur. defined the stationary phase as endcapped octadecylsilyl silica gel for chromatography $R$ $(5 \mu \mathrm{m})$ having size of $150 \times 4.6 \mathrm{~mm}$. On the other hand, according to the knowledge database [34], a HYB column is recommended by the Ph. Eur., but other stationary phases in compliance with the SST can be also used for this analysis. As mentioned, the SST requires a minimum peak-to-valley $(p / v)$ ratio of 1.2 for the impurity $\mathrm{G}$ and lamotrigine. Thus, for each stationary phase, this parameter was calculated. The obtained results confirmed that the SST value of 6.16 was calculated for the HYB column whereas the $p / v$ ratios ranged from 1.00 to 10.83 for other stationary phases studied (Table 3). Thus, an appropriate LC separation of lamotrigine and related compounds in accordance to the SST requirement was observed for 11 high-ranked stationary phases $(11 / 12 ; 91.67 \%)$, nine columns described by the $F_{\text {KUL }}$ values between 2 and $6(9 / 13 ; 69.23 \%)$, and none with low position in the KUL list ranking $(0 / 3 ; 0 \%)$. These results are similar to those previously found in the literature [18, 20-22, 24-28]. Moreover, the separation of lamotrigine at the concentration of $0.2 \mathrm{mg} / \mathrm{mL}$ in the presence of seven related compounds, each at the level of $0.1 \%$ of parent substance was performed using the stationary phases examined (Fig. 2). Next, for each column, the $t_{\mathrm{R}}$ and $R_{\mathrm{S}}$ for lamotrigine and its impurities were calculated, and these data are summarized in Table 3. Moreover, according to the $\mathrm{Ph}$. Eur. monograph, the $\% t_{\mathrm{RR}}$ parameters for parent substance and the related compounds A, E, F, and G were established. Notably, the $t_{\mathrm{R}}$ of the compounds of interest were longer for most stationary phases examined comparing to the reference column, while the shortest $t_{\mathrm{R}}$ values were noticed for NUA (no. 14) and ZSBA (no. 24). It should be also noticed that column nos. 13-15, 23, 24, and 26 were not in compliance with the second $\mathrm{Ph}$. Eur. recommendation (at least one analyte gave the $\% t_{\mathrm{RR}}$ values higher than $10 \%$ ). Thus, both $p / v$ parameters and the $\mathrm{Ph}$. Eur. recommendation related with the $t_{\mathrm{R}}$ of the analytes were realized by all columns classified as high-ranked in the KUL ranking list, while the probability of the appropriate LC separation of the compounds of interest significantly decreased according to increasing of the $F_{\mathrm{KUL}}$ parameter. It confirms that the column classification results provided by the KUL method and the column selectivity in separation of lamotrigine based on both the $\mathrm{Ph}$. Eur. requirements for the stationary phases examined were significantly correlated. 
Comparison based on the column selectivity in separation of lamotrigine and its related compounds using chemometric method

The evaluation of the KUL test procedure as a useful tool for predicting the appropriate stationary phases for the LC separation of lamotrigine in the presence of its impurities based on detailed description of the column test performance results was conducted using FA. For this assay, the $\% t_{R R}$ variables were omitted because the information provided by this variable is $100 \%$ redundant when the $t_{\mathrm{R}}$ of the analytes are taken into account. For this reason, the inverse of the matrix cannot be computed and the FA can basically not be performed or the FA is less reliable. Therefore, FA extracted from a standardized experimental data set of $t_{\mathrm{R}}$ and $R_{\mathrm{S}}$ of lamotrigine and the impurities of A, B, C, D, E, and G, as well as the SST test parameters established for the columns was calculated. The FA plots of variables and objects are presented in Fig. 4a and b, respectively. It should be noticed that here, the variance of the analyzed data involved in the variability of $t_{\mathrm{R}}$ of the analytes was mainly explained by the first PF1. These variables were found in cluster I, on the left side of the central part of the FA plot (Fig. 4a). The positions of the columns on the PF2 axes turned out to be related mainly to the variability of the $R_{\mathrm{SB}}, R_{\mathrm{S} \mathrm{G}}$, and $R_{\mathrm{S} \mathrm{A}}$ and then, the $p / v$ and $R_{\mathrm{S}}$ parameters. The variable of $R_{\mathrm{S} \text { B }}$ was found as an outlier on the bottom side of the graph, while the above-mentioned variables were located in cluster II on the right of the upper part of the plot, respectively. In summary, the first two PFs account for $65.92 \%$ of the data variability. Notably, most of the stationary phases positioned themselves within three clusters including columns 1-10,12, 13, 15, and 17 (cluster I); 16, 18, and 20-22 (cluster II); and 19, 23, 27, and 28 (cluster III), respectively (Fig. 4b). Fourteen columns forming cluster I were suitable for the LC determination of lamotrigine along with their impurities A-G according to the SST test. The second Ph. Eur. recommendation was not complied for stationary phases 13 and 15, which were found separately on the left side of cluster I. All columns located in cluster II offered also appropriate separations of the compounds of interest according to both $\mathrm{Ph}$. Eur. requirements. For them, medium and higher SST values were calculated, whereas the intermediate values of $t_{\mathrm{R}}$ and $R_{\mathrm{S}}$ of the analytes were observed. Contrary to them, insufficient $p / v$ parameters were established for the columns positioned within cluster III. Two stationary phases, nos. 14 and 24, were found close to each other on the right of the plot, while columns 11 and 26 were located on the bottom side of the graph. Among them, the column nos. 14 and 24 gave shorter $t_{\mathrm{R}}$ of the analyzed substances, while these values for the NUN (no. 11) were the longest, respectively. On the other hand, only NUA column gave an appropriate separation of lamotrigine and the impurity $\mathrm{G}$, but the $\mathrm{Ph}$. Eur. recommendation related with the $t_{\mathrm{RR}}$ of the analytes was not realized. Nevertheless, it is worth emphasizing that there are visible similarities between the graphs illustrated in Figs. $3 b$ and $4 b$. Thus, most of the stationary phases included within cluster I and cluster II extracted from FA based on four chromatographic parameters (Fig. 3b), were also found in cluster I and II based on column test performance, respectively (Fig. 4b). Only positions of column nos. 15 and 17 were different (cluster II-Fig. 3b; cluster I-Fig. 4b, respectively). The column nos. $19,23,27$, and 28 offering insufficient LC separation of the compounds of interest were positioned in cluster III, while the stationary phases, nos. 14 and 24, were found close to each other in both FA plots. On the other hand, different localization of XBSH column was also noticed (cluster IV-Fig. 3b; cluster I-Fig. 4b). Some inconsistencies were also observed for column nos. 11 and 26. These stationary phases were found close to each other in Fig. 3b, while they were positioned separately in the bottom part of the FA plot in Fig. 4b. Despite this, the localizations of 23 columns were consistent in both FA plots. It confirmed that the KUL method identified correctly most of the stationary phases examined. This fact allows to conclude that the usage of column classification system based on the KUL test procedure increases the probability of selecting columns that will be similar to and dissimilar from a reference stationary phase. Moreover, according to FA plots illustrated in Figs. 3a$4 \mathrm{~b}$, it can be noticed that the graphical visualization of the variables and the objects in the space created by the first PFs gives the possibility to perform the comparative study based on clearer and more in-depth interpretation of the obtained results. The results also confirmed that the stationary phases based on the type-B silica were the most suitable for the LC analysis of lamotrigine in the presence of its impurities when the lowest probability of an appropriate separation of the tested analytes was calculated for the columns containing embedded polar functional groups or polar end-capping.

\section{Conclusions}

This paper focuses on an application of the column classification system based on the KUL method in a selectivity study involving lamotrigine and its related impurities, using a set of 28 stationary phases. In the method proposed by Hoogmartens et al., each column is characterized by four chromatographic parameters which allow the calculation of the $F_{\mathrm{KUL}}$ value versus the reference stationary phase, following which the ranking list of the columns is built. The suitability of the columns examined was verified by the calculation of the SST according to the Ph. Eur. monograph and the chemometric assay of the column test performance. In summary, the $F_{\mathrm{KUL}}$ values provided by the KUL method 
and the positions of the 28 stationary phases studied, as specified by the KUL characteristics on FA plot were significantly correlated with the localization of stationary phases on the FA graph extracted from the retention parameters of the analytes indicating in detail each real pharmaceutical analysis. Thus, the results confirmed that the probability of appropriate column selection for the given analysis is higher after using the KUL test procedure. Moreover, an application of multivariate data processing, i.e., FA can be considered as valuable starting point for improving the reliable evaluation of the column classification system in pharmaceutical practice.

Acknowledgments The work was supported by the National Science Centre Project N N405 024340.

Open Access This article is distributed under the terms of the Creative Commons Attribution License which permits any use, distribution, and reproduction in any medium, provided the original author(s) and the source are credited.

\section{References}

1. Euerby MR, Petterson P (2000) A classification of commercially available RPLC columns - a tool for rational selection. LC-GC Europe 13:665-677

2. Snyder LR (2012) Efficient HPLC method: development and personal reflections. LC GC Europe 25:437-444

3. Abraham MMH (1993) Application of solvation equations to chemical and biochemical processes. Pure Appl Chem 65:2503-2515

4. Cruz E, Euerby MR, Johnson CM, Hackett CA (1997) Chromatographic classification of commercially available reversed-phase HPLC columns. Chromatographia 44:151-161

5. Claessens HA, van Straten MA, Cramers CA, Jezierska M, Buszewski B (1998) Comparative study of test methods for reversed-phase columns for high-performance liquid chromatography. J Chromatogr A 826:135-156

6. Snyder LR, Dolan JW, Carr PW (2004) The hydrophobicsubtraction model of reversed-phase column selectivity. J Chromatogr A 1060:77-116

7. Bączek T, Kaliszan R, Novotńa K, Jandera P (2005) Comparative characteristics of HPLC columns based on quantitative structureretention relationships (QSRR) and hydrophobic-subtraction model. J Chromatogr A 1075:109-115

8. Van Gyseghem E, Jimidar M, Sneyers R, De Smet M, Verhoeven E, Vander Heyden Y (2006) Stationary phases in the screening of drug/impurity profiles and in their separation method development: identification of columns with different and similar selectivities. J Pharm Biomed Anal 41:751-760

9. Jandera P, Novotná K (2006) Characterization of high-pressure liquid chromatography columns using chromatographic methods. Anal Lett 39:2095-2152

10. Buszewski B, Kowalska S, Kowalkowski T, Rozpędowska K, Michel M, Jonsson T (2007) HPLC columns partition by chemometric methods based on peptides retention. J Chromatogr B 845:253-260

11. Andries JPM, Claessens HA, Vander Heyden Y, Buydens LMC (2009) Strategy for reduced calibration sets to develop quantitative structure-retention relationships in high-performance liquid chromatography. Anal Chim Acta 652:180-188
12. Héberger K (2010) Sum of ranking differences compares methods or models fairly, Trends Anal. Chem 29:101-109

13. Héberger K, Škrbić B (2012) Ranking and similarity for quantitative structure-retention relationship models in predicting Lee retention indices of polycyclic aromatic hydrocarbons. Anal Chim Acta 716:92-100

14. Plenis A, Konieczna L, Miękus N, Bączek T (2013) Development of the HPLC method for simultaneous determination of lidocaine hydrochloride and tribenoside along with their impurities supported by the QSRR approach. Chromatography 76:255-265

15. Johnson AR, Johnson CM, Stoll DR, Vitha MF (2012) Identifying orthogonal and similar phase liquid chromatography stationary phases using the system selectivity cube and the hydrophobic subtraction model. J Chromatogr A 1249:62-82

16. Lesellier E (2012) Extension of the carotenoid test to superficially porous $\mathrm{C} 18$ bonded phases, aromatic ligand types and new classical C18 bonded phases. J Chromatogr A 1266:34-42

17. Dehouck P, Visky D, Van den Bergh G, Haghedooren E, Adams E, Kerner A, Vander Heyden Y, Massart DL, Kovács Z, Noszál B, Hoogmartens J (2004) Facilitated column selection in reversedphase liquid chromatography for pharmaceutical separations. LCGC Europe 17:592-601

18. Dehouck P, Visky D, Vander Heyden Y, Adams E, Kovács Z, Noszál B, Massart DL, Hoogmartens J (2004) Characterisation of reversedphase liquid-chromatographic columns by chromatographic tests. Comparing column classification based on chromatographic parameters and column performance for the separation of acetylsalicylic acid and related compounds. J Chromatogr A 1025:189-200

19. Haghedooren E, Kerner A, Noszál B, Hoogmartens J, Adams E (2007) Application of an improved column characterization system to evaluate the within and between batch variability. J Pharm Biomed Anal 44:634-639

20. Visky D, Haghedooren E, Dehouck P, Kovács Z, Kóczián K, Noszál B, Hoogmartens J, Adams E (2006) Facilitated column selection in pharmaceutical analyses using a simple column classification system. J Chromatogr A 1101:103-114

21. Kóczián K, Haghedooren E, Dragovic S, Noszál B, Hoogmartens J, Adams E (2007) Column selection for pharmaceutical analyses based on a column classification using four test parameters. J Pharm Biomed Anal 44:894-905

22. Haghedooren E, Németh T, Dragovic S, Noszál B, Hoogmartens J, Adams E (2008) Comparison of two column characterization systems based on pharmaceutical applications. J Chromatogr A 1189:59-71

23. Dragovic S, Haghedooren E, Nemeth T, Palabiyik IM, Hoogmartens J, Adams E (2009) Evaluation of two approaches to characterise liquid chromatographic columns using pharmaceutical separations. J Chromatogr A 1216:3210-3216

24. Plenis A, Balakowska E, Bączek T (2011) The comparison of two column classification systems during the chromatographic analysis of steroids. J Sep Sci 34:3310-3321

25. Németh T, Haghedooren E, Noszál B, Hoogmartens J, Adams E (2008) Three methods to characterize reversed phase liquid chromatographic columns applied to pharmaceutical separations. J Chemometr 22:178-185

26. Haghedooren E, Diana J, Noszál B, Hoogmartens J, Adams E (2007) Classification of reversed phase columns based on their selectivity towards vancomycin compounds. Talanta 71:31-37

27. Szulfer J, Plenis A, Bączek T (2012) Evaluation of a column classification method using the separation of alfuzosin from its related substances. J Chromatogr A 1229:198-207

28. Szulfer J, Plenis A, Bączek T (2012) Application of a column classification method in a selectivity study involving caffeine and its related impurities. Talanta 99:492-501

29. European Pharmacopoeia (2011) 7th ed., Council of Europe, Strasbourg, France 
30. Vervoort RJM, Derksen MWJ, Debets AJJ (1997) Monitoring of new silica-based reversed-phase stationary phases for the liquid chromatographic analysis of basic pharmaceuticals using principal components analysis. J Chromatogr A 765:157-168

31. Euerby ME, Petersen P (2003) Chromatographic classification and comparison of commercially available reverse-phase liquid chromatographic columns using principal component analysis. J Chromatogr A 994:13-36
32. West C, Lesellier E (2012) Chemometric methods to classify stationary phases for achiral packed column supercritical fluid chromatography: classification of columns for achiral SFC. J Chemometr 26:52-65

33. Plenis A, Olędzka I, Bączek T (2013) Classification of LC columns based on the QSRR method and selectivity toward moclobemide and its metabolites. J Pharm Biomed Anal 78-79:161-169

34. Ph. Eur. Knowledge. Database http://extranet.edqm.eu/publications/ recherches_sw.shtml 